TAUP2403-97

\title{
Gravitational Instanton for Black Hole Radiation
}

\author{
S. Massar ${ }^{a}$ and R. Parentani ${ }^{b}$ \\ a Raymond and Beverly Sackler Faculty of Exact Sciences, \\ School of Physics and Astronomy, Tel-Aviv University, Tel-Aviv 69978, Israel \\ ${ }^{b}$ Laboratoire de Mathématique et Physique Théorique, C.N.R.S. UPRES A 6083, \\ Faculté des Sciences, Université de Tours, 37200 Tours, France
}

\begin{abstract}
Hawking radiation is derived from the existence of a euclidean instanton which lives in the euclidean black hole geometry. Upon taking into account the gravitational field of the instanton itself, its action is exactly equal to one quarter the change in the horizon area. This result also applies to the Schwinger process, the Unruh process, and particle creation in deSitter space. The implications for horizon thermodynamics are discussed.
\end{abstract}

The thermodynamic properties of black holes were established more than 20 years ago. They are based on the identification of one quarter the horizon area with an entropy [1], the classical laws of black hole mechanics [2] and the process of black hole evaporation [3]. Since then much work has been devoted to obtain a better understanding of their origin, their implications, and the possible consequences of the gravitational back reaction. Hawking radiation has been derived in several different ways which all rely to some extent on regularity of the field state at the horizon, either in the Lorentzian space time, or in its euclidean continuation. In the latter case one obtains the Hartle-Hawking state characteristic of eternal black holes. The euclidean continuation of the black hole geometry has also been the subject of much interest since the work of Gibbons and Hawking [4] who interpreted it 
as the saddle point contribution to the partition function of quantum gravity.

In this Letter we shall show that Hawking radiation can be derived from the existence of a euclidean instanton. A similar instanton can also be associated to the Schwinger process (5] and pair creation in deSitter space [6]. The instanton is a periodic, static trajectory which lives in the euclidean continuation of space time. The exponential of minus its action yields the leading contribution to the probability to emit a particle. The most interesting aspect of the present analysis is to include the gravitational field of the instanton itself to obtain a self consistent euclidean solution. The Einstein-matter action is then reexpressed in terms of boundary terms only [7] [8], whereupon the action of the self consistent solution is equal to one quarter of the change of the black hole, the Rindler, or the deSitter horizon area. Thus the rate of particle production is

$$
R=N e^{-S_{E}}=N e^{\Delta A_{H} / 4}
$$

$(G=\hbar=c=1)$ where $N$ is a phase space factor. We note that this expression was first obtained in the context of pair production of black holes in an electric field [9] and of pair creation of black holes in de Sitter space [10]. In these cases one must also include on the r.h.s. of eq. (四) the area of the horizon of the produced black holes. It was pointed out in [8] that eq. (10) applies to the Schwinger process. And Eq. (11) was derived in the context of black hole evaporation in [1], both in a semiclassical calculation performed in a Lorenztian geometry and in the context of superstring theory, and it was argued that eq. (11) probably takes into account gravitational backreaction effects neglected in the background field approximation used by Hawking.

Before turning to the black hole problem, we first consider the Schwinger process in Minkowski coordinates to show that the instanton action is the leading contribution to the pair creation process. We then describe it in Rindler coordinates. The mapping to the black hole problem will then be straightforward because of the close analogies between Rindler coordinates in flat space and Schwarzshild coordinates in the black hole geometry.

The classical trajectory of a particle of mass $m$ and charge $e$ in a constant electric field 
$E$ is

$$
t(s)=t_{0}+\frac{1}{a} \operatorname{sh}(a m s) \quad z(s)=z_{0}+\frac{1}{a} \operatorname{ch}(a m s)
$$

where $a=e E / m$ is the acceleration. The corresponding euclidean trajectory is obtained by taking $s \rightarrow i s:$

$$
t(s)=t_{0}+\frac{i}{a} \sin (a m s) \quad z(s)=z_{0}+\frac{1}{a} \cos (a m s)
$$

This euclidean orbit naturally arises in the 5 th time formalism to derive the Schwinger pair production. It also appears upon evaluating the solution of the Klein-Gordon equation in a WKB approximation [12]. In second quantization, the probability not to produce pairs decreases as $\left|\left\langle 0_{\text {out }} \mid 0_{i n}\right\rangle\right|^{2}=e^{-\Gamma T V}$. In the 5 th time formalism, the rate of pair production per unit volume and unit time $\Gamma$ is expressed as $\Gamma T V=\operatorname{Im} \int d x \int_{0}^{\infty} \frac{d s}{s} \int \mathcal{D} x e^{i S(x, s)}$ where the path integral is over all periodic paths in time $s$ weighted by the action $S=\int d s \frac{1}{4}\left(\dot{t}^{2}-\right.$ $\left.\dot{z}^{2}\right)-e A_{t} \dot{t}+m^{2}$ where $A_{t}=E z$. In the above we have taken the transverse momentum (with respect to the electric field) to vanish. It can easily be taken into account by replacing $m^{2}$ by $m^{2}+k_{\perp}^{2}$, and integrating at the end of the calculation over $k_{\perp}$, see [12. The path integral can be evaluated by saddle point (which for constant $E$ is exact) to yield $\Gamma T V=$ $\operatorname{Im} \int d x \int_{0}^{\infty} \frac{d s}{s} \frac{e^{i S_{\text {class }}(s)}}{\sqrt{V V(s)}}$ where $S_{\text {class }}$ is the action to complete a closed trajectory evaluated on classical periodic trajectories with period $s$. The integral over proper time is entirely dominated by the poles of the VanVleck determinant $(V V)$ which occur at the values of $s=i 2 \pi n / a m(n=. .,-1,0,1, .$.$) corresponding to n$ windings along the euclidean trajectory eq. (3). Their classical action is $S=i \pi n m^{2} / e E$. Taking also into account the residue of the poles yields Schwinger's result $\Gamma=\left(\frac{E}{2 \pi}\right)^{2} \ln \left(1+e^{-\pi M^{2} / E}\right)$.

We now consider the same orbits in Rindler coordinates $(\rho, \tau)$ defined by $t=\rho s h \tau$ and $z=\rho c h \tau$ whereupon the metric is $d s^{2}=-\rho^{2} d \tau^{2}+d \rho^{2}$ and the EM potential in the boost invariant gauge is $A_{\tau}=E \rho^{2} / 2$. The euclidean orbits can be derived from the euclidean action $S_{E}=\int d s \frac{1}{4}\left(g_{\tau \tau} \dot{\tau}^{2}+g_{\rho \rho} \dot{\rho}^{2}\right)-e \dot{\tau} A_{\tau}+m^{2}$ (obtained from the Lorentzian action by taking $s \rightarrow i s$ and $\tau \rightarrow i \tau)$ where $g_{\tau \tau}=\rho^{2}$ and $g_{\rho \rho}=1$. It is convenient to reexpress $S_{E}$ in a Hamiltonian decomposition as $S_{E}=\int d s\left(\nu \dot{\tau}+p \dot{\rho}-H_{E}\right)$ where the euclidean hamiltonian is 


$$
H_{E}=g^{\tau \tau}\left(\nu+e A_{\tau}\right)^{2}+g^{\rho \rho} p^{2}-m^{2}
$$

The instanton, that is the static euclidean trajectory corresponding to a centered euclidean orbit eq. (3) with $t_{0}=z_{0}=0$, is obtained from the staticity conditions $p=0$, $\dot{p}=-\partial_{\rho} H_{E}=0$, the mass shell condition $H_{E}=0$ and the time evolution $\dot{\tau}=\partial_{\nu} H_{E}$. Explicitly it reads $\rho=1 / a, \tau=e E s$ and has energy $\nu=m^{2} / e E$. The periodicity of the euclidean solution results now from the topology of the euclidean continuation of Rindler space which is a plane described in polar coordinates. Regularity of the space at the origin imposes that $\tau$ (the polar coordinate) be periodic with period $2 \pi$. The action for one period is simply $S_{E}=\nu \Delta \tau=\pi m^{2} / e E$. It equals the action selected by the first pole $(n=1)$ of the Van Vleck.

We now introduce the electric and gravitational field of the instanton, whereupon we will find that $S_{E}$ is equal to one quarter the change in the area of the acceleration horizon. In order to control the self interactions of the instanton it is necessary that it no longer describes a point particle (due to its gravitational field a point particle becomes a black hole), but an extended object. The end result is independent of the nature of the extended object. For definiteness we take it to be a sheet which extends in the transverse direction. Inserting this in the Einstein-Maxwell action -expressed in a Hamiltonian decompositionyields

$$
\begin{aligned}
S_{E}= & \int d \tau\left(\int_{\Sigma_{\tau}} p^{i j} \partial_{\tau} g_{i j}+E^{i} \partial_{\tau} A_{i}+p \partial_{\tau} \rho\right. \\
& \left.\left.-N \mathcal{H}-N^{i} \mathcal{H}_{i}-N A_{\tau}\left(\operatorname{div} E-J^{\tau}\right)\right)\right)+S_{\text {Boundary }}
\end{aligned}
$$

where $\mathcal{H}$ and $\mathcal{H}_{i}$ are the hamiltonian and momentum constraints respectively. The boundary terms arise because one requires that the variation of $S_{E}$ with the physical boundary data fixed yield the equations of motion. In the present case the boundary conditions which are kept fixed are the asymptotic metric and electric field at infinity, and the vanishing of the laps at the horizon. This latter condition implies that the boundary term at the horizon is equal to one quarter the (infinite) area of the Rindler horizon. The euclidean action of the 
instanton is obtained by subtracting from $S_{E}$ the action without the shell present, but with the same boundary data at infinity. Because of this condition, the staticity of the instanton and the constraints, the action of the instanton is given by the (finite) difference of the boundary terms at the horizon only

$$
S_{E}(\text { instanton })=\Delta A_{H} / 4
$$

To prove that $S_{E}=\Delta A_{H} / 4$ behaves like an entropy we must consider a situation of thermal equilibrium. We therefore analyze the equilibrium of a uniformly accelerated detector with the Unruh heat bath [13]. The detector model we use is that of a "two level ion" in an electric field. By two level ion we mean a particle of mass $m$ and charge $e$ which can make a transition, by emitting a massless chargless quantum, to an excited state of rest mass $m^{\prime}$. In a constant electric field pairs of ions can be produced, in either the ground or excited state, with probability $P_{\text {pair } m}=e^{-\pi m^{2} / e E}$ and $P_{\text {pair } m^{\prime}}=e^{-\pi m^{\prime 2} / e E}$ respectively. On the other hand such a two level ion in an electric field behaves like a Unruh detector since it can make transitions between its internal states by absorbing or emitting a Rindler quantum. In [14] it was shown that the rates $\left(R_{m \rightarrow m^{\prime}}\right.$ and $\left.R_{m^{\prime} \rightarrow m}\right)$ for the Unruh process are related to the probability for the Schwinger process by the exact relation (obtained by taking into account corrections in $\left(m^{\prime}-m\right) / m$ and $\left.m / a\right)$ :

$$
\frac{R_{m \rightarrow m^{\prime}}}{R_{m^{\prime} \rightarrow m}}=\frac{P_{\text {pair } m^{\prime}}}{P_{\text {pair } m}}=e^{-\left(S_{E}\left(m^{\prime}\right)-S_{E}(m)\right)}
$$

(which can be understood by noting that the amplitudes describing the Schwinger and Unruh processes are related one to another by level crossing and CPT [14]). Unruh's result, namely that the detector perceives a thermal bath at temperature $T=a / 2 \pi$, is recovered in the (canonical) limit $m^{\prime}-m<<m$ whereupon $S_{E}\left(m^{\prime}\right)-S_{E}(m)=\left(m^{\prime}-m\right) \partial_{m} S_{E}(m)$. Indeed direct differentiation yields $\partial_{m} S_{E}(m)=\partial_{m} \pi m^{2} / e E=2 \pi / a$. The origin of this canonical result can be understood by recalling that $S_{E}=\Delta \tau \nu$ and by expressing $\nu$ as $\nu(m, e, \rho)=g_{\tau \tau}(\rho)^{1 / 2} m-e A_{\tau}(\rho)$ which results from the mass shell condition $H_{E}=0$, whereupon $\partial_{m} S_{E}(m)=\Delta \tau\left(\partial_{m} \nu+(d \rho / d m) \partial_{\rho} \nu\right)=\Delta \tau \partial_{m} \nu=\Delta \tau g_{\tau \tau}^{1 / 2}$ since $\partial_{\rho} \nu=0$ follows 
from the staticity condition $\partial_{\rho} H_{E}=0$. Thus $\partial_{m} S_{E}(m)$ is indeed the proper time to wind once round the orbit.

When the two level ion is in thermal equilibrium with the Unruh heat bath the probability to find it in the ground or excited state is given by $P_{m^{\prime}} / P_{m}=R_{m \rightarrow m^{\prime}} / R_{m^{\prime} \rightarrow m}$. By virtue of eq. (17) and eq. (11) this is also equal to

$$
\frac{P_{m^{\prime}}}{P_{m}}=\exp \left(A_{H}(m) / 4-A_{H}\left(m^{\prime}\right) / 4\right)
$$

where we have used the fact that physical processes are always governed by area changes to rewrite $\Delta A_{H}(m) / 4-\Delta A_{H}\left(m^{\prime}\right) / 4=A_{H}(m) / 4-A_{H}\left(m^{\prime}\right) / 4$. with $A_{H}\left(m^{\left({ }^{\prime}\right)}\right)$ the area of the horizon with a detector of mass $m^{\left({ }^{\prime}\right)}$ present. Eq. (8) is identical to that which would be obtained if the detector and the horizon form a microcanonical ensemble [14.

We now turn to the black hole problem. In this letter we shall only exhibit the periodic, stationary orbits living in the euclidean black hole geometry. In a future publication we intend to report on the relation between these orbits and more conventional derivations of black hole radiation. We first consider the emission of charged massive particles by a charged black hole. It was shown by Gibbons [15] that the evaporation of such a black hole is indeed controlled by the Schwinger process rather than the Hawking process. Therefore the preceding formalism should apply with minor modifications to this case.

The metric for such a charged black hole is $d s^{2}=-\left(1-2 M / r+Q^{2} / r^{2}\right) d t^{2}+(1-2 M / r+$ $\left.Q^{2} / r^{2}\right)^{-1} d r^{2}+r^{2} d \Omega^{2}$ and the EM potential regular at the horizon $r=r_{+}$is $A_{t}=Q / r-Q / r_{+}$. By making the change of variables $\rho=2 r_{+} \sqrt{r-r_{+}} / \sqrt{r_{+}-r_{-}}$the metric and EM potential reduce near the horizon to the flat space problem in Rindler coordinates. The action which yields the euclidean trajectories, obtained from the Lorentzian by taking $s \rightarrow i s, t \rightarrow i t$, is

$$
S_{E}=\int d s \frac{1}{4}\left(g_{t t} \dot{t}^{2}+g_{r r} \dot{r}^{2}\right)-e \dot{t} A_{t}-m^{2}
$$

where $g_{\mu \nu}$ is the euclidean metric. We have taken the angular momentum of the particle to vanish. It can easily be reeinstored by adding to $m^{2}$ the term $l^{2} / r^{2}$. The determination of the static euclidean orbit and of its action is obtained in the hamiltonian formalism exactly as 
in the electric case. The action of the euclidean orbit is $S_{E}=\beta \nu$ where $\beta$ in the periodicity of the euclidean time and $\nu$ the energy of the instanton. If the parameters $m, e, M, Q$ are such that the instanton lies in the region near the horizon where the correspondence with Rindler space obtains, then $S_{E}($ instanton $) \simeq \pi m^{2} / e E$ with $E \simeq Q / r_{+}^{2}$ the electric field at the horizon, as in the Schwinger process.

Our aim however is to make contact with the Hawking process per se. A possible procedure would be to take the charge $Q$ to zero in the above problem. In this case the euclidean static orbit disappears (this difficulty plagued an early attempt to apply the 5th time formalism to black hole evaporation [16]). In order to keep a static orbit as $Q \rightarrow 0$, one can either consider a particle with angular momentum, whereupon there is a static orbit at a finite distance from the horizon (for massless particles this is the well known orbit at $r=3 M)$ whose euclidean action for one period is once more $S_{E}=\beta \nu$. Or, for a particle with zero angular momentum one can adopt the following indirect procedure. We consider a Schwarzshild black hole surrounded at a very large distance $r_{0}$ by a pair of spherically symmetric condenser plates. The geometry of this configuration is $d s^{2}=-\gamma(r)(1-2 m(r) / r) d t^{2}+(1-2 m(r) / r)^{-1} d r^{2}+r^{2} d \Omega^{2}$ with $m\left(r<r_{0}-\Delta\right)=m_{B H}$, $m\left(r>r_{0}+\Delta\right)=m_{B H}+m_{C}$ and $\gamma\left(r>r_{0}+\Delta\right)=1, \gamma\left(r<r_{0}-\Delta\right)=O\left(m_{C} / r_{0}\right)$ where $m_{B H}$ is the black hole mass, $m_{C}$ is the energy of the condenser plates and $\Delta$ is the separation between the plates. The electromagnetic potential is $A_{t}\left(r<r_{0}-\Delta\right)=0$, $A_{t}\left(r>r_{0}+\Delta\right)=\phi=c s t$. Moreover the electric field between the condenser plates is taken to be such that it exactly counterbalances the gravitational attraction for a particle of mass $m$ and charge $e: E=\partial_{r} A_{t} \simeq M m / e r_{0}^{2}$. With this configuration such a particle will have a static orbit between the two condenser plates. The euclidean continuation of this orbit is the instanton for the Hawking process. Its action $S_{E}$ tends to $\beta m$ as $r_{0} \rightarrow \infty$, corresponding to the probability for a Schwarzshild black hole to emit a particle of mass $m$ which reaches infinity with zero kinetic energy.

It may seem surprising that this instanton is located at $r=\infty$ whereas black hole evaporation is usually associated with the existence of a horizon. But the existence of the 
horizon is crucial in the above calculation since it imposes the periodicity of the euclidean geometry in imaginary time. On the other hand the Hawking temperature, ie. the surface gravity, is determined by the whole geometry between the horizon and $r=\infty$. Together these combine to yield for the euclidean action the value $S_{E}=\beta m$ expected for Hawking radiation.

The inclusion of the gravitational and electric field of the instanton proceeds exactly as in eq. (5). The action of the static matter-gravitational configuration can be expressed as boundary terms only. The action of the instanton itself is obtained by subtracting the action of the black hole with the same mass and charge at infinity, but with the instanton absent. Whereupon one obtains that $S_{E}=\Delta A_{H} / 4$. The equality of $S_{E}=\Delta A_{H} / 4$ and $S_{E}=\beta \nu$ for infinitesimal $m$ and $e$ is a reexpression of the first law of black hole mechanics $\Delta A_{H} / 4=\beta \int_{\Sigma} \Delta T_{t}^{t}-A_{t} \Delta J^{t}$. This can be taken as an explanation of why Hawking radiation is consistent with the first law.

Particle production in deSitter space can be analyzed in similar manner. DeSitter space in static coordinates possesses a horizon at $r=\sqrt{3 / \Lambda}$. Regularity of the horizon in euclidean continuation implies that the euclidean time is periodic with period $\beta=2 \pi \sqrt{3 / \Lambda}$. A massive particle possesses a static, periodic, euclidean orbit at the origin whose action is $m \beta$ corresponding to the probability to produce a particle of mass $m$. The gravitational field of the instanton can be included as above and expressed as the change of the area of the deSitter horizon.

As for the Schwinger process, one can also consider two particles of neighboring masses which can make transitions from one mass state to the other thereby behaving like a particle detector. Then, following eq. (17), we conjecture that the probability for the detector to make a transition is given by the ratio of the probabilities for the production of the particle in its excited and ground state $P_{m^{\prime} \rightarrow m} / P_{m \rightarrow m^{\prime}}=e^{-\left(S_{E}\left(m^{\prime}\right)-S_{E}(m)\right)}$. As in the detector case, $A_{H} / 4$ then behaves like the entropy of the macroscopic system with which the detector is in thermal equilibrium. When the energy difference between detector states is small then a first order expansion of the difference $S_{E}(m)-S_{E}\left(m^{\prime}\right)$ is legitimate and one recovers the 
notion of temperature. The inverse temperature is given by the proper time to make one orbit $\partial_{m} S_{E}(m)=\beta \sqrt{g_{t t}}$ as in the electric case.

Recently, Jacobson [17] showed that the identification of horizon area with an entropy and of inverse acceleration with a temperature, together with the first law of thermodynamics, imply Einstein's equations. It is most remarkable that the two ingredients used by Jacobson can be derived from the (quantum) relation eq. (11). It is also important to note the connection between eq. (11) and the holographic hypothesis [18] wherein it is postulated that all the degrees of freedom inside a given volume are encoded in the surface surrounding the volume. Here it is the processes which occur inside, or outside, the volume which are encoded in the surface surrounding it. These results show the fundamental rôle causal horizons play in the quantum theory of gravity.

We would like to thank Y. Aharonov, R. Brout, F. Englert, S. Itzhaki, S. Nussinov, Ph. Spindel and Y. Zanelli for stimulating discussions. 


\section{REFERENCES}

[1] J. D. Bekenstein, Phys. Rev. D 7 (1973) 2333; Phys. Rev. D 9 (1974) 3292

[2] J. M. Bardeen, B. Carter and S. W. Hawking, Comm. Math. Phys. 31 (1973) 161

[3] S. W. Hawking, Comm. Math. Phys. 43 (1975) 199

[4] G. W. Gibbons and S. W. Hawking, Phys. Rev. D 15 (1977) 2752

[5] J. Schwinger, Phys. Rev. 82 (1951) 664

[6] G. W. Gibbons and S. W. Hawking, Phys. Rev. D 15 (1977) 2738

[7] M. Banados, C. Teitelboim and J. Zanelli, Phys. Rev. Lett. 72 (1994) 957

[8] S. W. Hawking and G.T. Horowitz, Class. Q. Grav. 13 (1996) 1487

[9] S. W. Hawking, G.T. Horowitz and S. Ross, Phys. Rev. D 51 (1995) 4302

[10] R. Bousso and S. W. Hawking, Phys. Rev. D 52 (1995 5659

[11] E. Keski-Vakkuri and P. Kraus, Microcanonical D-branes and Back Reaction, hepth/9610045

[12] R. Brout, R. Parentani and Ph. Spindel, Nucl. Phys. B 353 (1991) 209

[13] W. G. Unruh, Phys. Rev. D 14 (1976) 870

[14] R. Parentani and S. Massar, "Schwinger effect, Unruh effect and the production of accelerated black holes", hep-th/9603057

[15] G. W. Gibbons, Comm. Math. Phys. 44 (1975) 245

[16] R. Parentani and R. Brout, Nucl. Phys. B 388 (1992) 474

[17] T. Jacobson, Phys. Rev. Lett. 75 (1995) 1260

[18] G. 't Hooft, gr-qc/9310006; L. Susskind, J. Math. Phys.36 (1995) 6377 